\title{
A Novel Collateral Imaging Method Derived from Time-Resolved Dynamic Contrast-Enhanced MR Angiography in Acute Ischemic Stroke: A Pilot Study
}

\author{
(D)H.G. Roh, DE.Y. Kim, (DI.S. Kim, (D)H.J. Lee, (D).J. Park, DS.B. Lee, (D).W. Choi, DY.S. Jeon, DM. Park, DS.U. Kim, and (D)H.J. Kim
}

\begin{abstract}
BACKGROUND AND PURPOSE: Assessment of the collateral status has been emphasized for appropriate treatment decisions in patients with acute ischemic stroke. The purpose of this study was to introduce a multiphase MRA collateral imaging method (collateral map) derived from time-resolved dynamic contrast-enhanced MRA and to verify the value of the multiphase MRA collateral map in acute ischemic stroke by comparing it with the multiphase collateral imaging method (MRP collateral map) derived from dynamic susceptibility contrast-enhanced MR perfusion.
\end{abstract}

MATERIALS AND METHODS: From a prospectively maintained registry of acute ischemic stroke, MR imaging data of patients with acute ischemic stroke caused by steno-occlusive lesions of the unilateral ICA and/or the Ml segment of the MCA were analyzed. We generated collateral maps using dynamic signals from dynamic contrast-enhanced MRA and DSC-MRP using a Matlab-based in-house program and graded the collateral scores of the multiphase MRA collateral map and the MRP collateral map independently. Interobserver reliabilities and intermethod agreement between both collateral maps for collateral grading were tested.

RESULTS: Seventy-one paired multiphase MRA and MRP collateral maps from 67 patients were analyzed. The interobserver reliabilities for collateral grading using multiphase MRA or MRP collateral maps were excellent (weighted $\kappa=0.964$ and 0.956 , respectively). The agreement between both collateral maps was also excellent (weighted $\kappa=0.884$; 95\% confidence interval, 0.819-0.949).

CONCLUSIONS: We demonstrated that the dynamic signals of dynamic contrast-enhanced MRA could be used to generate multiphase collateral images and showed the possibility of the multiphase MRA collateral map as a useful collateral imaging method in acute ischemic stroke.

ABBREVIATIONS: AIS = acute ischemic stroke; DCE-MRA = dynamic contrast-enhanced MRA; DSC-MRP = dynamic susceptibility contrast-enhanced MR perfusion; $\mathrm{mMRA}=$ multiphase MRA; MRP $=$ MR perfusion

$\mathbf{T}$ he cerebral collaterals are alternative vascular channels for maintaining blood perfusion to the ischemic brain distal to an arterial occlusion. The collateral status varies among patients with

Received March 4, 2019; accepted April 12.

From the Departments of Radiology (H.G.R., J.W.C.), Neurology (J.J.P.), and Neurosurgery (Y.S.J.), Konkuk University Medical Center, Konkuk University School of Medicine, Seoul, Korea; Department of Radiology (E.Y.K.), Gachon University Gil Medical Center, Incheon, Korea; Siemens Healthineers Ltd (I.S.K.), Seoul, Korea; Departments of Neurosurgery (H.J.L., S.U.K.), Neurology (S.B.L.), and Radiology (H.J.K.), Daejeon St. Mary's Hospital, The Catholic University of Korea, Daejeon, Korea; and Department of Radiology (M.P.), Gangnam Severance Hospital, Yonsei University College of Medicine, Seoul, Korea.

This study was supported by the National Research Foundation of Korea as the subject No. NRF-2017R1A2B1008020.

Please address correspondence to Hyun Jeong Kim, MD, PhD, Department of Radiology, Daejeon St. Mary's Hospital, Medical School, The Catholic University of Korea, 64 Daeheung-Ro, Jung-Gu, Daejeon, 34943, Korea; e-mail: mskendy@cmcdj.or.kr

- Indicates open access to non-subscribers at www.ajnr.org

http://dx.doi.org/10.3174/ajnr.A6068 acute ischemic stroke (AIS) and determines the final infarct size and clinical outcome. ${ }^{1}$ Prior studies of endovascular treatment showed that better collaterals are associated with less infarct growth and better functional outcome, whereas poor collaterals are linked to hemorrhagic complications and poor recanalization rates. ${ }^{2-4}$ In terms of endovascular treatment in patients beyond 5 hours from onset, it was suggested that a better collateral status could extend the time window. ${ }^{5-9}$ Therefore, assessment of the collateral status has been emphasized for appropriate treatment decisions in patients with AIS.

The imaging methods for collateral assessment have progressed from single-phase to multiphase imaging. ${ }^{10-13}$ The arterial scoring method based on single-phase CTA had a critical limitation of underestimating the leptomeningeal collaterals with a longer transit time due to early triggering of a static acquisition. ${ }^{10}$ A method based on multiphase CTA overcame this limitation but still has problems, including radiation exposure, use of iodine contrast media, and imperfect prediction of infarct volume. An- 


\begin{tabular}{|c|c|c|c|c|c|}
\hline \multirow[b]{2}{*}{ Parameter } & \multicolumn{5}{|c|}{ Acute Stroke MRI } \\
\hline & SS EPI-DWI & SWI & DCE-MRA (TWIST) & DSC-MRP & T2-FLAIR \\
\hline TR/TE (ms) & $4800 / 71$ & $28 / 20$ & $2.62 / 0.95$ & $1600 / 30$ & $9000 / 95$ \\
\hline $\mathrm{TI}(\mathrm{ms})$ & & & & & 2500 \\
\hline Turbo factor & & & & & 21 \\
\hline Flip angle & & $15^{\circ}$ & $21^{\circ}$ & $90^{\circ}$ & $150^{\circ}$ \\
\hline Bandwidth (Hz/pixel) & 1672 & 120 & 780 & 1446 & 206 \\
\hline Slice thickness/gap (mm) & $5 / 2$ & $2 / 0$ & $1.2 / 0$ & $5 / 2$ & $5 / 2$ \\
\hline FOV $(\mathrm{mm})$ & $240 \times 240$ & $240 \times 195$ & $400 \times 300$ & $240 \times 240$ & $240 \times 217$ \\
\hline Matrix size (mm) & $130 \times 130$ & $384 \times 156$ & $320 \times 182$ & $128 \times 128$ & $256 \times 174$ \\
\hline B-value (s/mm²) & 0,1000 & & & & \\
\hline Measurements & 1 & 1 & 30 & 60 & 1 \\
\hline GRAPPA & 2 & 2 & $3 \times 2$ & & 2 \\
\hline Temporal resolution (sec) & & & 1.6 & 1.6 & \\
\hline Dynamic reconstruction mode & & & Forward share & & \\
\hline$K$-space sampling (center/periphery) $(\%)$ & & & $15 / 20$ & & \\
\hline
\end{tabular}

Note:-SS indicates single-shot; TWIST, time-resolved imaging with stochastic trajectories; GRAPPA, generalized autocalibrating partially parallel acquisition.

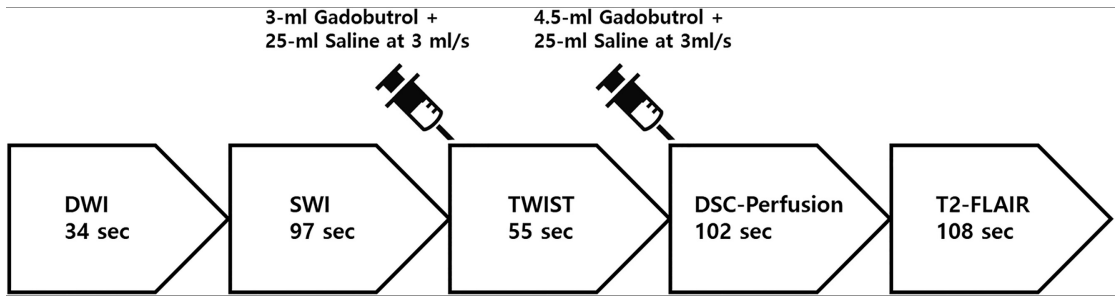

FIG 1. MR imaging protocol for AIS diagnosis. contralateral ICA and/or MCA; 2) patients with intracranial hemorrhage on admission MR imaging; 3) patients with previous major stroke with a residual disability (modified Rankin Scale score, $\geq 2$ ); 4) patients with other brain lesions, such as neoplasm, infection, or demyelination, and so forth; 5) patients unable to undergo MR imaging; and 6) patients with poor-quality MR imaging due to

other multiphase collateral imaging method (collateral flow map) derived from dynamic susceptibility contrast-enhanced MR perfusion (DSC-MRP) was introduced, and the study showed that the collateral flow map had prognostic value in AIS with a major artery occlusion. ${ }^{12}$ However, DSC-MRP does not provide angiographic information, which is an indispensable imaging parameter for endovascular treatment. Dynamic contrast-enhanced MRA (DCE-MRA) is also an angiographic imaging method, and like DSC-MRP, it detects intracranial dynamic signals according to the flow of MR imaging contrast media. We developed a new multiphase collateral imaging method, (the mMRA collateral map), using the dynamic signals of DCE-MRA. This study was conducted to verify the value of the mMRA collateral map as a collateral imaging method by comparing it with the multiphase collateral imaging method derived from DSC-MRP (MRP collateral map).

\section{MATERIALS AND METHODS \\ Patients}

We analyzed MR imaging data of patients with AIS from a prospectively maintained registry. The MR imaging data of consecutive patients from September 2015 to March 2017 at 2 university medical centers were analyzed retrospectively. Inclusion criteria for this study were as follows: 1) patients who presented with symptoms consistent with AIS within 8 hours of symptom onset; 2) patients older than 18 years of age; 3 ) patients who underwent brain MR imaging, including DWI, DSC-MRP, and DCE-MRA, at admission; and 4) patients with occlusion or severe stenosis ( $\geq 70 \%)$ of the unilateral ICA and/or M1 segment of the MCA (M1 MCA) and the associated symptoms. Exclusion criteria were as follows: 1) patients with occlusion or severe stenosis of the various artifacts. Institutional review boards of Konkuk University Medical Center and Daejeon St. Mary's Hospital approved this study. All participants provided informed consent.

\section{Imaging Protocol and Postprocessing to Generate the Collateral Map}

All MR imaging examinations were performed on a 3T Magnetom Skyra MR system (Siemens, Erlangen, Germany) with a 20channel head/neck coil. The routine MR imaging protocol for AIS included DWI, SWI, DCE-MRA, DSC-MRP, and FLAIR. The acquisition parameters are summarized in Table 1. DCE-MRA was performed by the repetitive acquisition of coronal 3D imaging slabs extending from the aortic arch to the cranial vertex, including the whole brain, with a temporal resolution of 1.6 seconds per dynamic acquisition. 3D rotational arteriography was reconstructed with a dataset of an arterial time point of DCE-MRA to interpret the arterial status. The total acquisition time for the MR imaging was 6 minutes 36 seconds, and the total amount of contrast medium was $7.5 \mathrm{~mL}$ of gadobutrol (Gadovist; Bayer Schering Pharma, Berlin, Germany) (Fig 1).

We developed a Matlab-based program (MathWorks, Natick, Massachusetts) to produce an mMRA collateral map and an MRP collateral map (Fig 2). The sequential postprocessing steps are as follows: 1) opening and reading DICOM source images of DCEMRA and DSC-MRP; 2) cropping all DICOM images of DCEMRA to obtain only the brain region by removing the cervical and aortic arch areas, which are not necessary for the mMRA collateral map; 3) storing all acquired DCE-MRA or DSC-MRP image data in a 4D matrix; 4) creating MIP images for each time point that represent the bolus passage for each phase after subtraction of the 


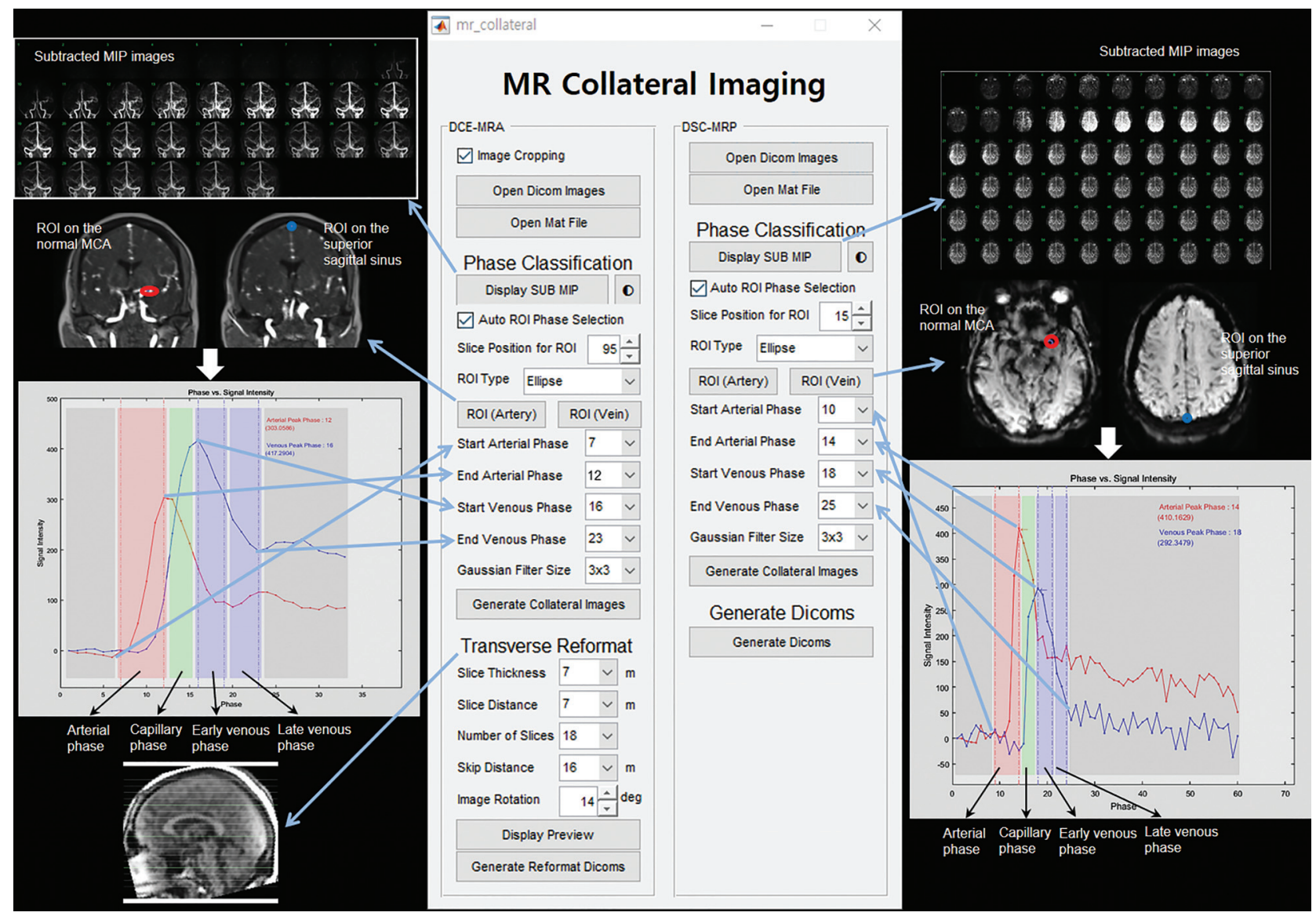

FIG 2. Postprocessing sequences for the mMRA collateral map (left) and the MRP collateral map (right). After being loaded and stored in a 4D matrix of DICOM source images of DCE-MRA and DSC-MRP for approximately 1 minute, subtracted MIP images with both the time and spatial information are displayed. We apply ROIs on the MCA in nonischemic hemisphere and superior sagittal sinus. Arterial and venous signal intensity-time curves are generated by plotting signal changes in the ROIs, and 4 phases (arterial phase = from the beginning of arrival of the contrast in the MCA to the arterial bolus peak; capillary phase = from just past the arterial bolus peak to just before the venous bolus peak in the superior sagittal sinus; early venous phase $=$ first half of the venous phase from the venous bolus peak to the starting point of the venous plateau; late venous phase $=$ second half of the venous phase) of collateral maps are determined according to the signal intensity-time curves automatically. In mMRA collateral maps, an axial reformatting process for the original coronal images is added before generating the collateral map.

first phase noncontrast image from each subsequent time-series image to eliminate the background signal; 5) obtaining arterial and venous signal intensity-time curves for ROIs on the MCA in nonischemic hemisphere and the superior sagittal sinus by plotting signal changes in the ROIs; 6) separating the 4D image sets from DCE-MRA and DSC-MRP into the following 4 phases according to bolus passage status, which were plotted on the signal intensity-time curve (arterial phase = from the beginning of arrival of the contrast in the MCA to the arterial bolus peak; capillary phase $=$ from just past the arterial bolus peak to just before the venous bolus peak in the superior sagittal sinus; early venous phase $=$ first half of the venous phase from the venous bolus peak to the starting point of the venous plateau; late venous phase $=$ second half of the venous phase); and 7) reformatting the $4 \mathrm{D}$ image sets from DCE-MRA and DSC-MRP into averaged 4-phase axial image sets (collateral map) with the desired reconstruction parameters (slice range covering the whole brain, slice thickness ranging from 7 to $15 \mathrm{~mm}$, slice distance, and number of slices) (Figs 3 and 4). For coronally acquired DCE-MRA data, additional reconstruction processing was needed to obtain the axial images parallel to the anterior/posterior commis- sure line. The postprocessing times from loading of source images of DCE-MRA and DSC-MRP to generating the mMRA collateral map and MRP collateral map were approximately 5 and 2 minutes, respectively.

\section{Collateral Grading and Statistical Analysis}

We developed a preliminary collateral grading system with a 6-point scale for collateral maps to define the collateral-perfusion status and severity of ischemia in the MCA territory by comparison with the unaffected cerebral hemisphere. The collateral grading system was defined as follows: $5=$ excellent, $4=$ good, $3=$ intermediate to good, $2=$ intermediate to poor, $1=$ poor, $0=$ very poor (Table 2 ). This grading system was made by modifications of 2 previously reported grading systems by Tan et al ${ }^{14}$ and Menon et al. ${ }^{7}$ To make the collateral grading easy and straightforward, we adopted the criterion of one-half of the MCA territory at each phase. Additionally, one-half of the MCA territory is widely considered the threshold above which a poor outcome is highly probable. $^{15}$

In visually undetermined, borderline cases with a collateralperfusion delay of approximately one-half of the MCA territory, 


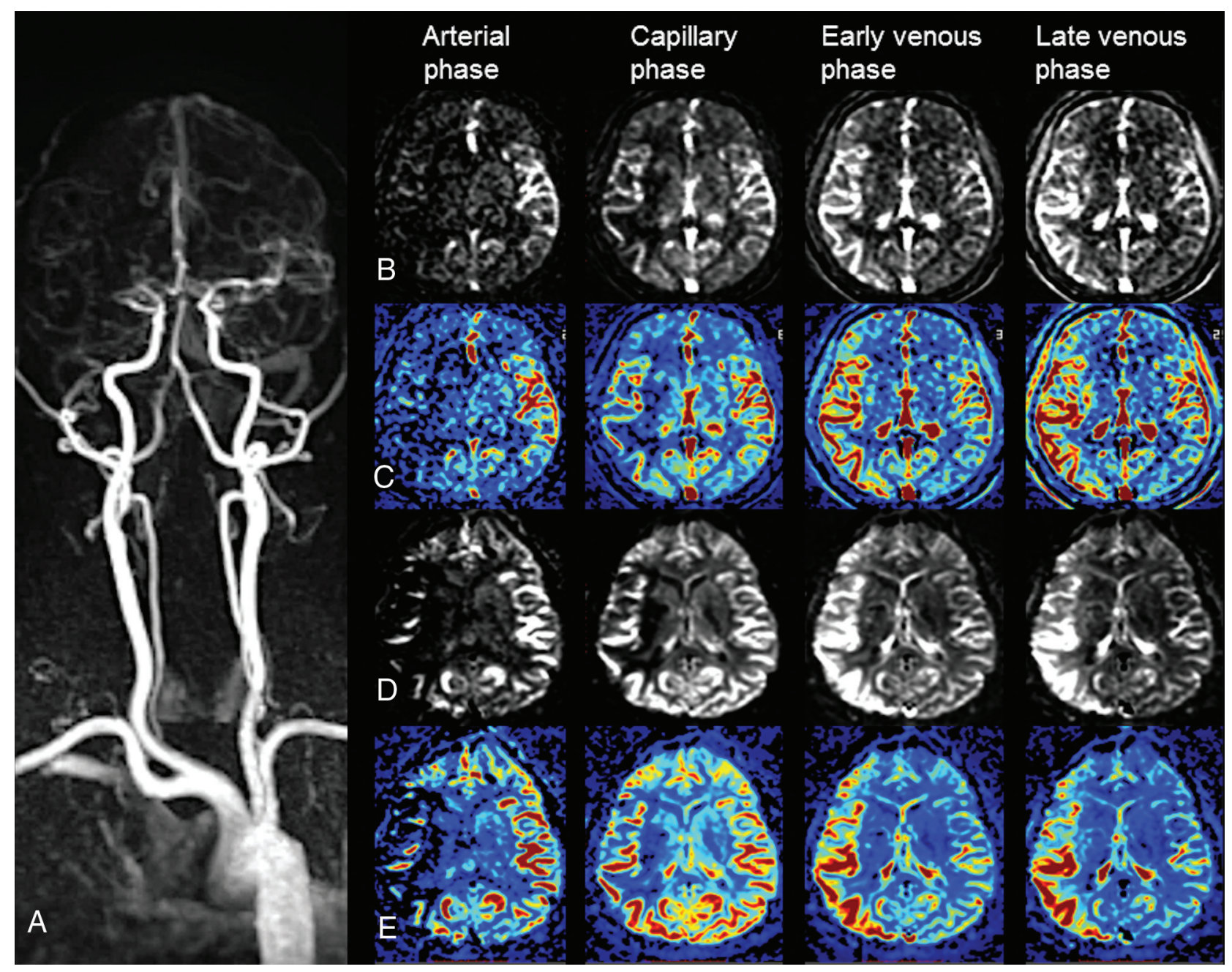

FIG 3. mMRA and MRP collateral maps at 2 hours 18 minutes and 2 hours 21 minutes after symptom onset in a 61-year-old woman with occlusion of the right MCA. The 3-month mRS score of the patient was 2. A, DCE-MRA shows occlusion of the right M1 MCA. Gray-scale (B) and color-coded $(C)$ mMRA collateral maps. On the mMRA collateral maps, the collateral-perfusion delay involves more than one-half of the MCA territory, including the subcortical structures in the capillary phase and no collateral-perfusion delay in the early venous phase. The collateral score is 3 (intermediate to good). Gray-scale (D) and color-coded (E) MRP collateral maps. The MRP collateral map shows the same collateral score as the mMRA collateral map.

the collateral score was decided by counting the number of regions with a collateral-perfusion delay similar to that of ASPECTS. ${ }^{16}$ The differentiation of 3 subcortical structures (eg, caudate, lentiform nucleus, and internal capsule) might be arbitrary or limited with the resolution of the collateral map, and subcortical structures are less influenced by collateral circulation; therefore, we divided the MCA territory into 8 areas: M1, M2, M3, M4, M5, M6, the insular ribbon, and the subcortical structures (Fig 5). For example, a collateral-perfusion delay greater than one-half of the MCA territory means that the collateral-perfusion delay involves $>4$ of the 8 regions in the MCA territory. Two experienced neuroradiologists (H.J.K. and H.G.R. with 15 years and 17 years of experience, respectively) independently graded the collateral scores of the mMRA and MRP collateral maps in a blinded fashion on 2 separate occasions 1 week apart. Both neuroradiologists were also blinded to all clinical and other imaging data. To assess the intermethod agreement between the mMRA collateral map and the MRP collateral map, we determined the final scores by consensus. The functional outcome of patients was assessed at day 90 with mRS. A favorable outcome was defined as an $\mathrm{mRS}$ of $\leq 2$, or an mRS equal to the prestroke $\mathrm{mRS}$ if the prestroke $\mathrm{mRS}$ was $>2$, at day 90 .

Statistical analysis was performed using SPSS Statistics, Version 21 (IBM, Armonk, New York). The interobserver reliabilities for collateral grading of both collateral maps and the intermethod agreement between the mMRA and the MRP collateral maps were measured by the Cohen weighted $\kappa$. Coefficients were interpreted as follows: $\kappa=0.21-0.40$, fair agreement; $\kappa=0.41-0.60$, moderate agreement; $\kappa=0.61-0.80$, substantial agreement; $\kappa=0.81-$ 1.0 , almost perfect agreement.

\section{RESULTS}

Sixty-seven patients, including 34 women and 33 men, with a mean age of $69.7 \pm 11.5$ years (range, 38-93 years), met the inclusion criteria. All patients presented with AIS. Thirteen patients (19.4\%) presented with occlusion of the extracranial ICA, 7 patients $(10.4 \%)$ presented with severe stenosis of the extracranial ICA, 4 patients $(6.0 \%)$ presented with occlusion of the intracra- 


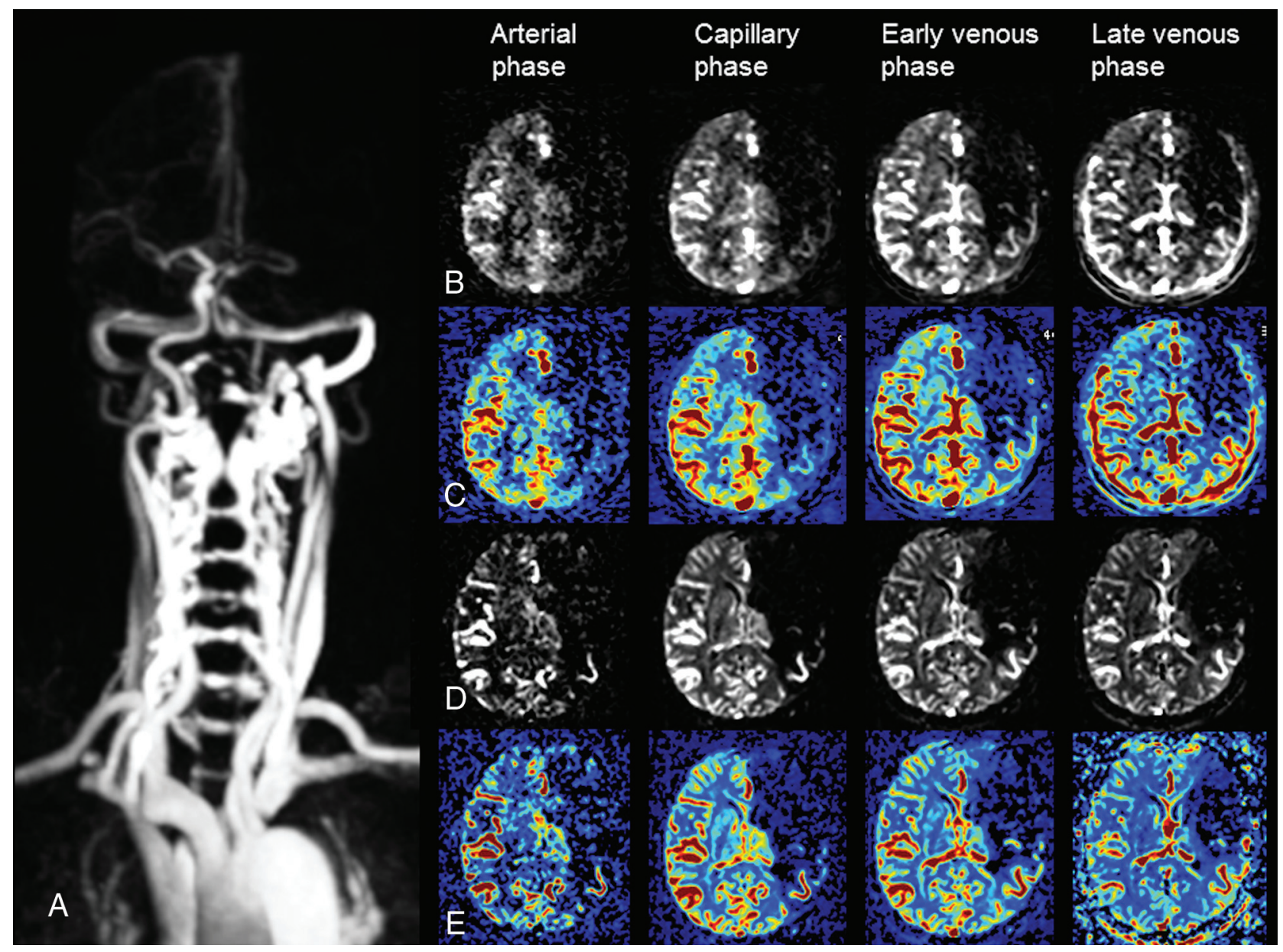

FIG 4. mMRA and MRP collateral maps at 5 hours 44 minutes and 5 hours 47 minutes after symptom onset in a 72 -year-old man with occlusion of the left ICA and MCA. This patient died 27 days after admission. A, DCE-MRA shows occlusion of the left proximal ICA and MCA. Gray-scale $(B)$ and color-coded $(C)$ mMRA collateral maps. On the mMRA collateral maps, the collateral-perfusion delay involves more than one-half of the MCA territory, including the subcortical structures in the capillary phase, and persists until the late venous phase. The collateral score is 0 (very poor). Gray-scale (D) and color-coded (E) MRP collateral maps. The MRP collateral map shows the same collateral score as the mMRA collateral map.

Table 2: Collateral grading system for analysis of the collateral map

\begin{tabular}{l|l}
\hline \multicolumn{1}{c}{ Score } & \multicolumn{1}{c}{ Description of Collateral Status } \\
\hline 5 (Excellent) & $\begin{array}{r}\text { No or small }{ }^{\text {a }} \text { collateral-perfusion delay in the MCA territory in the capillary phase regardless of the collateral } \\
\text { status in the arterial phase } \\
\text { Collateral-perfusion delay equal to or less than one-half of MCA territory in the capillary phase and no or } \\
\text { small delay in the early venous phase }\end{array}$ \\
3 (Intermediate to good) & $\begin{array}{r}\text { 1) Collateral-perfusion delay equal to or less than one-half of the MCA territory in the capillary phase and } \\
\text { equal or less than one-half in the early venous phase } \\
\text { 2) Collateral-perfusion delay more than one-half of the MCA territory in the capillary phase and no or small } \\
\text { delay in the early venous phase } \\
\text { Collateral-perfusion delay more than one-half of the MCA territory in the capillary phase and equal to or } \\
\text { less than one-half in the early venous phase } \\
\text { Collateral-perfusion delay more than one-half of the MCA territory in the early venous phase and equal to } \\
\text { or less than one-half in the late venous phase } \\
\text { Collateral-perfusion delay/defect more than one-half of the MCA territory in the late venous phase } \\
\text { regardless of perfusion status at previous phases }\end{array}$ \\
\hline (Poor) & (Very poor)
\end{tabular}

\footnotetext{
a "Small" indicates an area $<1$ of 8 MCA regions (Fig 5).
}

nial ICA, 2 patients $(3.0 \%)$ presented with severe stenosis of the intracranial ICA, 15 patients $(22.4 \%)$ presented with occlusion of the ICA and M1 MCA, 1 patient (1.5\%) presented with severe stenosis of the ICA and M1 MCA, 23 patients (34.3\%) presented with occlusion of the M1 MCA, and 2 patients (3.0\%) presented with severe stenosis of the M1 MCA. After good recovery ( $\mathrm{mRS} 0$ or 1) from the initial stroke during the study period, 4 of $67 \mathrm{pa}$ tients had a recurrent stroke $(n=2)$ or TIA $(n=2)$ due to occlusion of the left MCA $(n=1)$, severe in-stent restenoses at the left distal ICA $(n=1)$ and right MCA $(n=1)$, and persistent severe stenosis of the proximal left ICA $(n=1)$, respectively. Finally, 71 paired mMRA collateral maps and MRP collateral maps of 67 


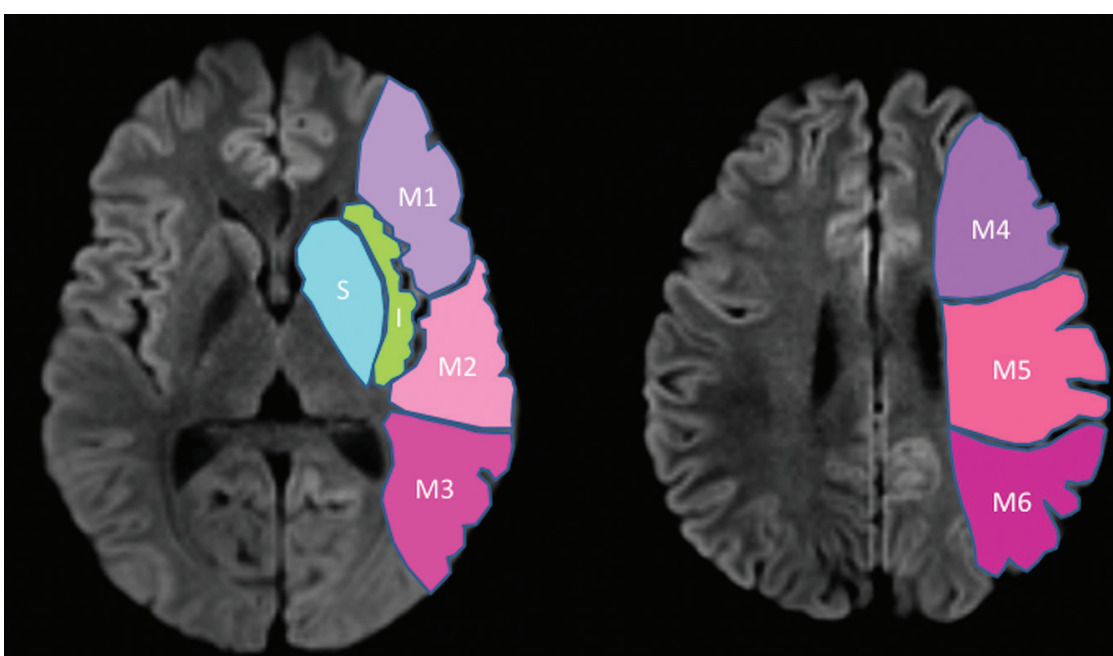

FIG 5. Eight topographic regions of the MCA territory for MR collateral imaging (collateral map). $\mathrm{I}=$ insular ribbon; $\mathrm{S}=$ subcortical structures (basal ganglia and internal capsule); $\mathrm{M1}, \mathrm{M} 2$, and $\mathrm{M} 3$ are the anterior, lateral, and posterior MCA territories, respectively, at the basal ganglia level; M4, $\mathrm{M} 5$, and M6 are the anterior, lateral, and posterior MCA territories, respectively, at the supraganglionic level.

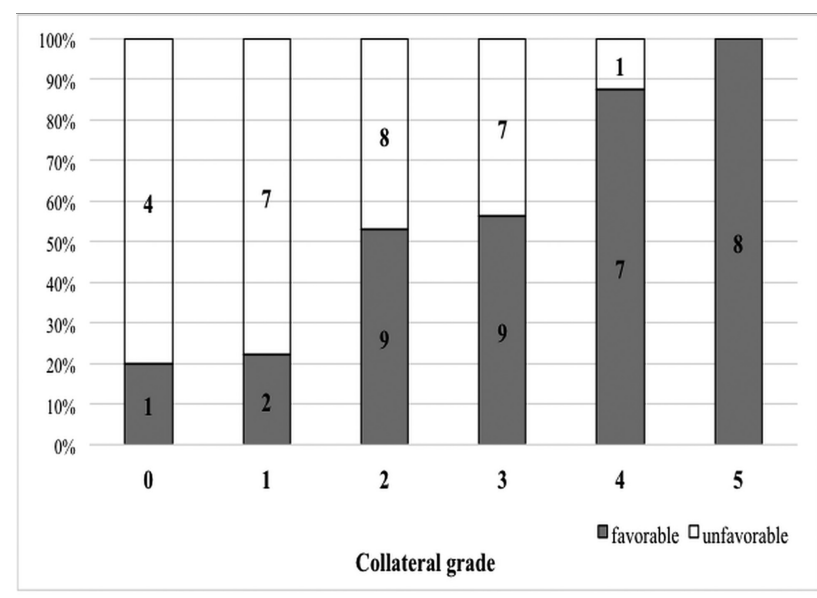

Numbers in columns are number of patients.

FIG 6. A $100 \%$ stacked column chart of the functional outcomes and collateral grades of patients.

patients were generated successfully and compared. The interobserver reliability for collateral grading of the mMRA collateral map (weighted $\kappa=0.964 ; 95 \%$ confidence interval, $0.929-0.999$ ) and of the MRP collateral map (weighted $\kappa=0.956$; $95 \%$ confidence interval, 0.919-0.993) was almost perfect. The agreement between both collateral maps was also excellent (weighted $\kappa=$ 0.884; 95\% confidence interval, 0.819-0.949) (Figs 3 and 4). Among 63 patients, excepting 4 patients with recurrent stroke or TIA, excellent collateral grade was found in 8 patients $(12.7 \%)$; good collateral grade, in $8(12.7 \%)$; intermediate to good collateral grade, in 16 (25.4\%); intermediate to poor collateral grade, in 17 (27.0\%); poor collateral grade, in 9 (14.3\%); and very poor collateral grade, in $5(7.9 \%)$. The functional outcome of the patients was demonstrated in Fig 6.

\section{DISCUSSION}

We demonstrated that the dynamic signals of DCE-MRA could be used to generate multiphase collateral images, called the mMRA collateral map, and showed good agreement between the mMRA collateral map and the MRP collateral map for the grading of collateral status. Kim et al reported that multiphase collateral images derived from DSC-MRP, a similar concept to our MRP collateral map, could provide information about collateral circulation and had prognostic value in 134 patients with AIS. ${ }^{12}$ Their collateral flow map consisted of early, mid-, and late phases divided according to reference time point and representing the midpoint of the midphase on the signal intensity-time curve, and the 3 phases were correlated with arterial, capillary, and venous phases on DSA, respectively. They showed good correlation between MRP- and DSA-based collateral grades. DCE-MRA is an angiographic imaging method, but the dynamic signals are essentially similar to those of DSC-MRP, which are produced by MR imaging contrast media during passage from the cerebral arteries to the dural sinuses. Therefore, we evaluated the value of the mMRA collateral map as a collateral imaging method by comparing it with the MRP collateral map.

Several studies have shown that a large ischemic core and poor collaterals among imaging parameters were strong predictors of an unfavorable response to endovascular treatment and poor functional outcome, and excluding patients with a large ischemic core and poor collateral circulation may avoid futile and dangerous recanalization therapy. ${ }^{2,5,6,8,9,17,18}$ Conversely, good collateral circulation can limit ischemic core expansion and prolong the time that penumbral tissue-at-risk remains salvageable until reperfusion therapy, and some studies have suggested that the time window for endovascular treatment can be successfully extended in patients with good collaterals. ${ }^{2,5,6,8,9,17,18}$ Therefore, a quick and accurate estimation of the collateral status is essential for endovascular treatment in patients with AIS. Multiphase CTA was recently used in the Endovascular Treatment for Small Core and Anterior Circulation Proximal Occlusion with Emphasis on Minimizing CT to Recanalization Times (ESCAPE) trial and was shown to help determine the clinical outcome. ${ }^{3,11}$ The multiphase CTA technique is quick and easy to use without complex postprocessing and acquires temporal information at 3 time points. ${ }^{11}$ However, the MR imaging-based collateral map has several fundamental advantages over multiphase CTA, in addition to the avoidance of radiation and iodine contrast media ${ }^{19}: 1$ ) The phases of the MR imaging-based collateral map are not fixed and can be individualized after obtaining time-intensity curves and delay correction. Therefore, the MR imaging-based collateral map may overcome intrinsic patient factors and problems due to scan timing and may reflect each phase more precisely. 2) MRP and MRA permit repeat scanning if the first scan is deemed inappropriate. 3) The MR imaging-based collateral map can provide more detailed information about the tissue-level, brain parenchymal collateral perfusion in addition to cortical artery information. 
Previous studies have shown that half-dose single-phase contrast-enhanced MRA and perfusion did not negatively affect either the image quality or the quantitative analysis of perfusion data. $^{20,21}$ Another study showed that time-resolved DCE-MRA with a low dose $(2-3 \mathrm{~mL})$ of gadobutrol could provide good image quality with a signal-to-noise ratio comparable with that of conventional single-phase 3D high-resolution contrast-enhanced MRA with a standard dose. ${ }^{22}$ Therefore, we could adopt a lowdose protocol for DCE-MRA, and the remaining contrast media could be used for DSC-MRP. We succeeded in generating mMRA and MRP collateral maps in all patients. Additional angiographic imaging is not necessary in the stroke MR imaging protocol when the mMRA collateral map is applied as collateral imaging, unlike the MRP collateral map, which shortens the imaging time while also requiring a minimal dose of contrast media.

We used a self-developed collateral grading system because there was no established grading method for 4-phase collateral imaging. Our grading system was largely based on that of multiphase CTA according to the perfusion lag and its extent. The differences were that we used the criterion of one-half of the MCA territory at each grading score, and we included parenchymal collateral perfusion as well as cortical arteries in comparison with the healthy contralateral MCA territory. Because an infarct volume of more than one-half of the MCA territory has been widely considered the threshold for poor outcome of acute MCA stroke, we thought that the one-half-area criterion could be optimal for visual assessment while avoiding ambiguous terms, such as "some," "a few," or "periphery," used in the existing grading systems. ${ }^{11,12,23}$ In borderline cases with collateral-perfusion delay in approximately one-half of the MCA territory, the 8-region MCA diagram was used to resolve the ambiguity in collateral grading. We divided the venous phase into early venous phase and late venous phase because we observed that patients with a larger area of collateral-perfusion delay/defect in the late venous phase tended to have a large ischemic core and a worse prognosis than patients with a smaller area. We considered that validation of the clinical meaning of early and late venous phases was necessary in the application of the collateral map to patients with AIS.

Our study has several limitations. First, the clinical efficacy of the mMRA collateral map as a collateral imaging method was not evaluated. We showed a chart with a tendency for an increasing proportion of patients with favorable outcome as increasing collateral grade, but the numbers in these data are too small to evaluate statistically. Although the DSC-MRP-based collateral imaging has been evaluated clinically, the evaluation was a retrospective observational study at a single medical center. ${ }^{12}$ Further clinical studies are necessary to validate the clinical usefulness of the mMRA collateral map and the grading method. We are pursuing a prospective clinical study for this validation. Second, 5 additional minutes were needed for postprocessing to generate an mMRA collateral map. Therefore, the total imaging time was 11 minutes 36 seconds for the MR imaging acquisition and postprocessing in this study. Although this was an acceptable time for acute stroke imaging, further effort should be made to shorten the imaging time.

\section{CONCLUSIONS}

We introduced a new collateral imaging method derived from time-resolved DCE-MRA and showed that this method can provide collateral information, like the collateral imaging method derived from DSC-MRP. Future validation of the clinical usefulness of the mMRA collateral map and large clinical studies are needed.

Disclosures: Hyun Jeong Kim—RELATED: Grant: National Research Foundation of Korea, Comments: This study was supported by National Research Foundation of Korea, subject No. 2017R1A2B1008020.* *Money paid to the institution.

\section{REFERENCES}

1. Liebeskind DS. Collateral circulation. Stroke 2003;34:2279-84 CrossRef Medline

2. Liebeskind DS, Tomsick TA, Foster LD, et al; IMS III Investigators. Collaterals at angiography and outcomes in the Interventional Management of Stroke (IMS) III trial. Stroke 2014;45:759-64 CrossRef Medline

3. Goyal M, Demchuk AM, Menon BK, et al; ESCAPE Trial Investigators. Randomized assessment of rapid endovascular treatment of ischemic stroke. N Engl J Med 2015;372:1019-30 CrossRef Medline

4. Bang OY, Saver JL, Buck BH, et al; UCLA Collateral Investigators. Impact of collateral flow on tissue fate in acute ischaemic stroke. J Neurol Neurosurg Psychiatry 2008;79:625-29 Medline

5. Ribo M, Flores A, Rubiera M, et al. Extending the time window for endovascular procedures according to collateral pial circulation. Stroke 2011;42:3465-69 CrossRef Medline

6. Hwang YH, Kang DH, Kim YW, et al. Impact of time-to-reperfusion on outcome in patients with poor collaterals. AJNR Am J Neuroradiol 2015;36:495-500 CrossRef Medline

7. Menon BK, Qazi E, Nambiar V, et al; Interventional Management of Stroke III Investigators. Differential effect of baseline computed tomographic angiography collaterals on clinical outcome in patients enrolled in the Interventional Management of Stroke III Trial. Stroke 2015;46:1239-44 CrossRef Medline

8. Hill MD, Demchuk AM, Goyal M, et al; IMS3 Investigators. Alberta Stroke Program early computed tomography score to select patients for endovascular treatment: Interventional Management of Stroke (IMS)-III trial. Stroke 2014;45:444-49 CrossRef Medline

9. Bang OY, Saver JL, Kim SJ, et al. Collateral flow predicts response to endovascular therapy for acute ischemic stroke. Stroke 2011;42: 693-99 CrossRef Medline

10. Menon BK, Smith EE, Modi J, et al. Regional leptomeningeal score on CT angiography predicts clinical and imaging outcomes in patients with acute anterior circulation occlusions. AJNR Am J Neuroradiol 2011;32:1640-45 CrossRef Medline

11. Menon BK, d'Esterre CD, Qazi EM, et al. Multiphase CT angiography: a new tool for the imaging triage of patients with acute ischemic stroke. Radiology 2015;275:510-20 CrossRef Medline

12. Kim SJ, Son JP, Ryoo S, et al. A novel magnetic resonance imaging approach to collateral flow imaging in ischemic stroke. Ann Neurol 2014;76:356-69 CrossRef Medline

13. Parthasarathy R, Sohn SI, Jeerakathil T, et al. A combined arterial and venous grading scale to predict outcome in anterior circulation ischemic stroke. J Neuroimaging 2015;25:969-77 CrossRef Medline

14. Tan JC, Dillon WP, Liu S, et al. Systematic comparison of perfusion-CT and CT-angiography in acute stroke patients. Ann Neurol 2007;61:533-43 CrossRef Medline

15. Souza LC, Yoo AJ, Chaudhry ZA, et al. Malignant CTA collateral profile is highly specific for large admission DWI infarct core and poor outcome in acute stroke. AJNR Am J Neuroradiol 2012;33: 1331-36 CrossRef Medline

16. Barber PA, Demchuk AM, Zhang J, et al. Validity and reliability of a 
quantitative computed tomography score in predicting outcome of hyperacute stroke before thrombolytic therapy: ASPECTS Study Group-Alberta Stroke Programme Early CT Score. Lancet 2000; 355:1670-74 CrossRef Medline

17. Bang OY, Saver JL, Kim SJ, et al; UCLA-Samsung Stroke Collaborators. Collateral flow averts hemorrhagic transformation after endovascular therapy for acute ischemic stroke. Stroke 2011;42:2235-39 CrossRef Medline

18. Leng X, Fang H, Leung TW, et al. Impact of collateral status on successful revascularization in endovascular treatment: a systematic review and meta-analysis. Cerebrovasc Dis 2016;41:27-34 CrossRef Medline

19. Aviv RI, Parsons M, Bivard A, et al. Multiphase CT angiography: a poor man's perfusion CT? Radiology 2015;277:922-94 CrossRef Medline

20. Nael K, Meshksar A, Ellingson B, et al. Combined low-dose contrastenhanced MR angiography and perfusion for acute ischemic stroke at 3T: a more efficient stroke protocol. AJNR Am J Neuroradiol 2014; 35:1078-84 CrossRef Medline

21. Manka C, Träber F, Gieseke J, et al. Three-dimensional dynamic susceptibility-weighted perfusion MR imaging at 3.0 T: feasibility and contrast agent dose. Radiology 2005;234:869-77 CrossRef Medline

22. Bak SH, Roh HG, Moon WJ, et al. Appropriate minimal dose of gadobutrol for 3D time-resolved MRA of the supra-aortic arteries: comparison with conventional single-phase high-resolution 3D contrastenhanced MRA. AJNR Am J Neuroradiol 2017;38:1383-90 CrossRef Medline

23. Higashida RT, Furlan AJ, Roberts H, et al; Technology Assessment Committee of the American Society of Interventional and Therapeutic Neuroradiology, Technology Assessment Committee of the Society of Interventional Radiology. Trial design and reporting standards for intra-arterial cerebral thrombolysis for acute ischemic stroke. Stroke 2003;34:e109-37 Medline 